\title{
The essentiality of histo- and cytochemical studies of skeletal muscle in the investigation of
} neuromuscular disease

\author{
W. King Engel, M.D.
}

IN THE INVESTIGATION of neuromuscular disease, histologic examination of skeletal muscle biopsies is generally accepted as an important part both of routine diagnostic procedures and of more specialized research. The purpose of the present report is to establish that the use of histo- and cytochemical technics combined with light microscopy is also an essential aspect of both types of investigation. Substantiating evidence is presented in the form of 6 examples, chosen from recent studies, of cytopathologic changes in neuromuscular disease as demonstrated by these technics.

Two fundamental types of abnormalities in skeletal muscle are detectable with histoand cytochemical technics: (a) a generalized change of a given reaction, for example, loss of phosphorylase activity, and (b) a localized change of activity confined either to a specific type of muscle fiber (as in the new myopathy with rod-shaped structures) or to certain regions of individual muscle fibers (as in target fibers and central core disease). Further, if the cytochemical technics are sufficiently refined, they may be used to demonstrate the functional activity of fine structural components of the muscle cell. On the basis of such data, predictions can often be made as to the fine structural changes which electron-microscopy will reveal. It must be understood, though, that some cytochemical changes might not be accompanied by changes of fine structure and vice versa. Cytochemistry combined with electron-microscopy would give even more detailed information, but this approach has 2 major limitations: (a) only a very few cytochemical reactions are as yet refined enough to be used with electron-microscopy and (b) study of a large number of specimens is restricted by the technical difficulties encountered in electron-microscopy.

Although histochemical investigations of pathologic human muscle have been reported by other workers, ${ }^{1-6}$ the cytopathologic details were not emphasized.

\section{METHODS}

Skeletal muscle tissue was obtained by biopsy from patients suspected of having neuromuscular disease, most of whom were on the service of Dr. G. M. Shy. The biopsy specimen was rapidly frozen and from it 2 to $10 \mu$ thick serial sections were cut in a cryostat (figure 12). The unfixed sections were then stained by a number of histochemical procedures. ${ }^{7,8}$ Cellular morphology, including intracellular detail, was also demonstrated by the modified Gomori trichrome ${ }^{9}$ and the hematoxylin-eosin methods. Mitochondrial oxidative enzyme activity was studied by the reactions for succinate dehydrogenase, ${ }^{10}$ reduced diphosphopyridine-nucleotide (DPNH) dehydrogenase, ${ }^{11}$ DPN-linked lactate dehydrogenase, DPN-linked $\alpha$-glycerophosphate dehydro-

From the Medical Neurology Branch, National Institute of Neurological Diseases and Blindness, National Institutes of Hoalth, U. S. Public Health Service, Department of Health, Education and Welfare. Bethesda, Maryland.

Presented as the S. Weir Mitchell Award Essay at the fourteenth annual meeting of the American Academy of Neurology, New York City, April 26, 1962. 
genase, ${ }^{12}$ "menadione-linked" $\alpha$-glycerophosphate dehydrogenase, ${ }^{13}$ and cytochrome oxidase. ${ }^{14}$ The last step of each of the oxidative enzyme systems is considered to be located in mitochondria. Nitro Blue Tetrazolium was the indicator used with each of the dehydrogenase reactions. Constituents of the sarcoplasm studied cytochemically were pre-formed glycogen by the periodic acid-Schiff (PAS) technic, ${ }^{15}$ amylophosphorylase (phosphorylase), ${ }^{16}$ and, in some instances, uridine diphosphate glucose (UDPG)-glycogen transferase activity. ${ }^{17}$ The UDPG-glycogen transferase reaction was improved by the addition of uridine triphosphate (2 mg. per ml.). Adenosine triphosphatase (ATPase) activity, ${ }^{18}$ especially that of the myofibrils, was also studied. In one condition, central core disease, ${ }^{19}$ the transverse component of the sarcoplasmic reticulum was studied by the Holmes silver (argyrophile) technic.

\section{RESULTS AND DISCUSSION}

A. Normal cytochemistry. In cross-sections, reactions can be localized to the myofibrils (figure 32) or to the intermyofibrillar region (figures 14, 17, 23, 25, 33, 34, 39). The latter, stained or unstained, appears as a network (figure 8) around the myofibrils. Three of the components which lie in the intermyofibrillar region throughout the muscle fiber are the mitochondria (figure 25), the sarcoplasmic reticulum (figure 17), and the aqueous, nonmembranous sarcoplasm (figures 14, 23, 33, $34,39)$. In longitudinal sections, the mitochondrial oxidative enzyme activity frequently was localized as double transverse rows of dots or somewhat elongated forms at the level of the I-band, one row being on each side of the Z-band (figure 9). The argyrophilic material was located mainly in the intermyofibrillar region at the level of the A-band I-band junction (figure 18) and presumably represented the triads of the transverse component of the sarcoplasmic reticulum. ${ }^{20-22}$ The 3 sarcoplasmic reactions studied (preformed glycogen, phosphorylase, and UDPG-glycogen transferase) were localized in the intermyofibrillar region and on longitudinal section appeared in wide transverse bands at the level of the I-bands (figure 34). Under certain standard conditions (dried sections, $\mathrm{pH}$ 9.4) only the myofibrils showed ATPase activity (figure 32). The activity was confined to the A-band, excepting the $\mathrm{H}$-band portion. As the myofibrillar A-band ATPase had the same location as antigenic myosin (see Tunik and Holtzer, ${ }^{23}$ Engel and Horvath, 24 and their references), it was considered ${ }^{8}$ to represent the known myosin ATPase activity. Undried sections usually showed calcium-resistant "membranous" (sarcolemmal and intermyofibrillar) ATPase in addition to myofibrillar A-band activity. An unusual variation sometimes occurred in the wasp, ${ }^{25}$ with activity being present only in the mitochondria and the Z-bands (figure 11). There was no ATPase activity of the endomysial or perimysial connective tissue in normal, denervated, or myopathic muscle, in contrast to the observation by others ${ }^{3,6}$ of high ATPase activity in connective tissue of muscular dystrophy. All of these cytolocalizations are generally as expected ${ }^{8}$ from electronmicroscopic and biochemical data. Further, cytochemical studies ${ }^{25}$ of 2 special muscles, the toadfish swim bladder muscle (figure 10) and the wasp fibrillar flight muscle (figure II) (in each of which the intermyofibrillar components are more easily distinguishable with light microscopy than in human skeletal muscle), reveal cytolocalization to be as expected from electron-microscopy of these tissues. ${ }^{26,27}$

B. Normal histochemistry. For many decades it has been known that there are 2 major types of muscle fibers (figures $1-6,8$ ) in the striated muscle of many species (see $\mathrm{Knoll}^{28}$ and Krüger $\left.{ }^{29}\right)$. It is preferable that they be called "Type I" and "Type II" fibers, ${ }^{34}$ to avoid names with controversial implications (figure 8). Sometimes 3 types of muscle fibers occur (figure 7). The relationship of fiber type, structural as well as histochemical, to fiber diameter is not constant throughout the limb muscles. Histochemical differences (figures $1-6)$ in the degree of activity of the different fiber types are known. ${ }^{30-34}$ The reciprocal relationship in a given fiber of the degree of several mitochondrial oxidative enzyme activities (for example, DPNH dehydrogenase) and the degree of phosphorylase activity has been demonstrated ${ }^{33,34}$ in human and other skeletal muscle (figures 1,3 ; table 1). 
TABLE 1

CORRELATION OF HISTOCHEMICAL ACTIVITIES IN DIFFERENT TYPES OF MUSCLE FIBERS

\begin{tabular}{|c|c|c|}
\hline & Type I & Type II \\
\hline DPNH dehydrogenase & high & low \\
\hline DPN-linked lactate dehyclrogenase & high & Jow \\
\hline succinate dehydrogenase & high & low \\
\hline cytochrome oxidase & high & low \\
\hline benzidine-peroxidase & high & low \\
\hline $\begin{array}{l}\text { DPN-linked alpha-glycerophosphate } \\
\text { dehydrngenase }\end{array}$ & medium & medium \\
\hline $\begin{array}{l}\text { menadione-linked alpha-glycero- } \\
\text { phosphate dehydrogenase }\end{array}$ & low & high \\
\hline phosphorylas. & low & high \\
\hline myofibrillar A-band ATPase & low & high \\
\hline glycogen & low & high \\
\hline UDPG-glycogen transferase & low & high \\
\hline
\end{tabular}

DPNH dehydrogenase activity is greater in Type I fibers (figure 1) and phosphorylase is greater in Type II fibers (figure 3 ). Recently, the interesting reciprocal relationship between "menadione-linked" $\alpha$-glycerophosphate dehydrogenase and all the other oxidative enzymes studied (including DPN-linked $\alpha$-glycerophosphate dehydrogenase) was reported in the fibers of rat muscle. ${ }^{35}$ The reciprocal relationship between "menadionelinked" $\alpha$-glycerophosphate dehydrogenase and succinate, DPNH, and DPN-linked lactate clehydrogenases has been confirmed in human muscle in the present study (figures 1,4 ). However, the DPN-linked $\alpha$-glycerophosphate dehydrogenase seems to have an intermediate position, being more nearly equal (but in a different pattern of localization) in the different fiber types (figure 2). There is usually a parallel relationship between the amount of preformed glycogen and of phosphorylase activity in a given fiber, both in rat and human muscle ${ }^{8}$ (figures 3,5 ), in contrast to a previous report ${ }^{36}$ of a reciprocal relationship in the rat. In human muscle, the UDPGglycogen transferase activity, too, has usually a parallel relationship to phosphorylase in a given fiber, ${ }^{8}$ contrasting with the reciprocal relationship reported in the rat. ${ }^{36}$ The amounts of myofibrillar A-band ATPase and of phosphorylase show a parallel relationship in a given fiber of human muscle. ${ }^{8}$ The intrafusal fibers of a given muscle spindle also are of different histochemical types, but they will not be discussed here.

It is in reference to this summary of the normal histo- and cytochemical appearance of human skeletal muscle that the 6 examples of cytopathologic changes are to be presented.

C. Phosphorylase defect of skeletal muscle. later adult type. A 52-vear-old white female recently presented with progressive proximal muscle weakness and wasting of only three year's duration, without muscle cramps or myoglobinuria. Study of her muscle biopsy with the routine histochemical reactions revealed complete absence of phosphorylase $a$

Fig. 1. DPNH dehydrogenase, $225 \times$ (figures 1 to 6 are serial sections, same magnification). Normal muscle of 6-year-old girl, vastus lateralis. The activity is greater in Type I fibers.

Fig. 2. DPN-linked a-glycerophosphate dehydrogenase. The degree of activity is about equal in both types of fibers, but the pattern is different.

Fig. 3. Phosphorylase. The activity is greater in Type II fibers, a reciprocal pattern to that of figure 1 .

Fig. 4. "Menadione-linked" a-glycerophosphate dehydrogenase. The fibers of Type II show greater activity, producing a pattern reciprocal to that of figure 1 .

Fig. 5. PAS for pre-formed glycogen. The activity is greater in Type II fibers, a pattern parallel to that of figure 3 .

Fig. 6. Myofibrillar A-band ATPase. The fiber activity pattern is parallel to that of figure 3 , with the greater activity in Type II fibers.

Fig. 7. Phosphorylase, $89 \times$. Normal muscle, gastrocnemius. Note 3 degrees of fiber activity. The most reactive (darkest) fibers are smallest and the least reactive ones are largest. (More often, the opposite correlation of phosphorylase activity and fiber diameter occurs.)

Fig. 8. Modified trichrome, cross-section, $560 \times$. Normal. There is one light fiber of Type II surrounded by four dark ones of Type $I$. In the latter, the intermyofibrillar material is more darkly stained and forms a more complete network pattern than in the former. 

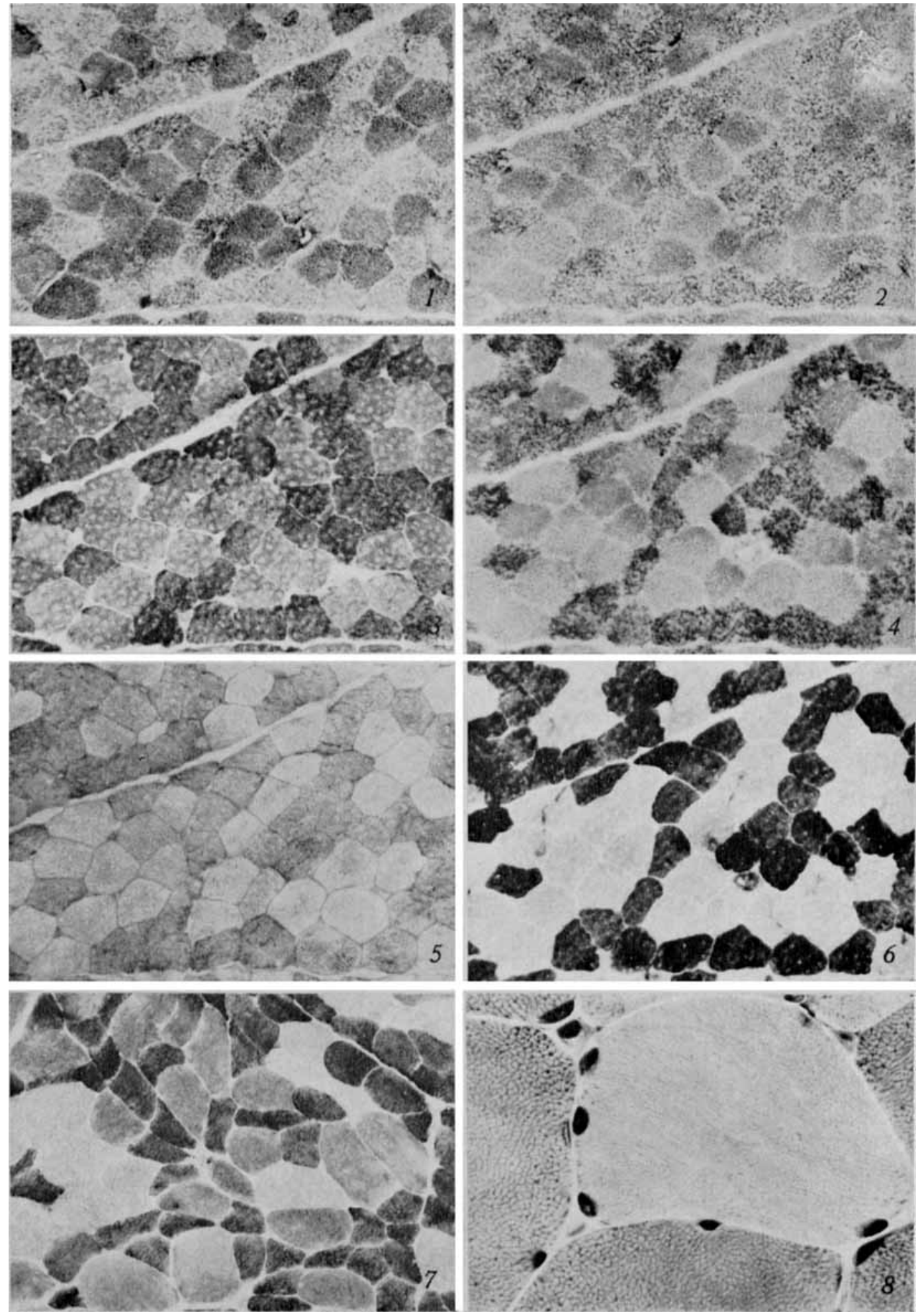

(All figures are cross-sections of fresh frozen human adult skeletal muscle tissue, unless otherwise noted) 
and $b$ in the skeletal muscle, with normal activity in the smooth muscle of the blood vessel walls (figure 13). UDPG-glycogen transferase activity was present in the skeletal muscle fibers histochemically. A second muscle biopsy examined histochemically and biochemically (Dr. Hibbard Williams) confirmed the phosphorylase defect. In addition to the generalized absence of phosphorylase in the biopsied skeletal muscle fibers, there were also focal architectural disturbances displayed by the other cytochemical reactions. These appeared as regions of increased or decreased concentrations of intermyofibrillar material (figures 14-16), as well as of more severe morphologic changes. It has not been determined whether or by what means the more generalized phosphorylase defect is responsible for the focal degenerative changes in some of the muscle fibers.

Previous examples of a disease associated with a phosphorylase defect of skeletal muscle have been published by McArdle, ${ }^{37}$ by Schmid, Mahler and colleagues, ${ }^{38-42}$ and by Pearson, Mommaerts, and colleagues. ${ }^{43-45}$ All of the 4 previously reported cases of "McArdle's disease" have been similar, following the stages suggested by Schmid and Hammaker: ${ }^{42}$

\section{Childhood and adolescence-easy muscle} fatigability, intermittent dark urine.

2. Early adult life-cramping muscle pain on exertion, followed by transient myoglobinuria.

3. Fourth (or fifth) decade-persistent and progressive weakness and wasting of muscle, absent or rare myoglobinuria.

The histochemical discovery of a skeletal muscle phosphorylase defect in the present case thus disclosed a new, unsuspected "adult" or "late onset" form of McArdle's syndrome. The present patient appears to have skipped the first 2 stages, but then presented with findings generally compatible with those of the other reported cases in their late stage. (The details of this case will be published elsewhere. ${ }^{40}$ )

The known forms of phosphorylase defect in skeletal muscle can be classified as follows:

Fig. 9. Succinate dehydrogenase, longitudinal section, polarized light, $1400 \times$. Normal muscle. The wide A-bands and intermediate thin Z-bands are brightly anisotropic. The dark dots indicating mitochondrial loci appear in the isotropic (dark) I-bands, frequently in pairs with one dot on each side of the Z-band.

Fig. 10. Phosphorylase, $560 \times$. Toadfish swim bladder muscle. The tubular muscle fibers have single (or occasionally multiple) central cores of aqueous sarcoplasmic material rich in phosphorylase. There may also be small collections of sarcoplasmic phosphorylase at the periphery of the myofibrillar tube, under the sarcolemma.

Fig. 11. "Membranous" ATPase, longitudinal section, $1400 \times$. Wasp fibrillar flight muscle. ATPase activity is demonstrated not in the A-bands but in the Z-bands and in mitochondria. Two large mitochondria are adjacent to each sarcomere. (Compare the size of mitochondria in figures 9 and 11.)

Fig. 12. Myofibrillar A-band ATPase, $560 \times$. The A-bands are darkly stained, the I-bands show no activity. The "contour-map" appearance of the muscle fiber is produced by thin $(2 \mu)$ cross-sectioning of misaligned myofibrils and is not pathologic.

Fig. 13. Phosphorylase, $225 \times$. Phosphorylase defect of skeletal muscle, later adult type (figures 13 to 16 ). The smooth muscle of the blood vessels contains abundant activity but the skeletal muscle fibers have none.

Fig. 14. PAS for pre-formed glycogen, $560 \times$. Some fibers have glycogen of normal amount and location (in the intermyofibrillar region), while in other fibers there are regions of reduced glycogen.

Fig. 15. DPN-linked lactate dehydrogenase, $225 \times$. Many fibers have regions of increased and decreased concentrations of activity (compare with figure 35). Foci of increased activity often occur under the sarcolemma. In some fibers, there are subsarcolemmal blebs lacking mitochondrial activity as well as myofibrillar material.

Fig. 16. Succinate dehydrogenase, $225 \times$. Regions of decreased and increased concentrations of activity occur in the fibers (compare with figure 14). There are a few subsarcolemmal blebs. 

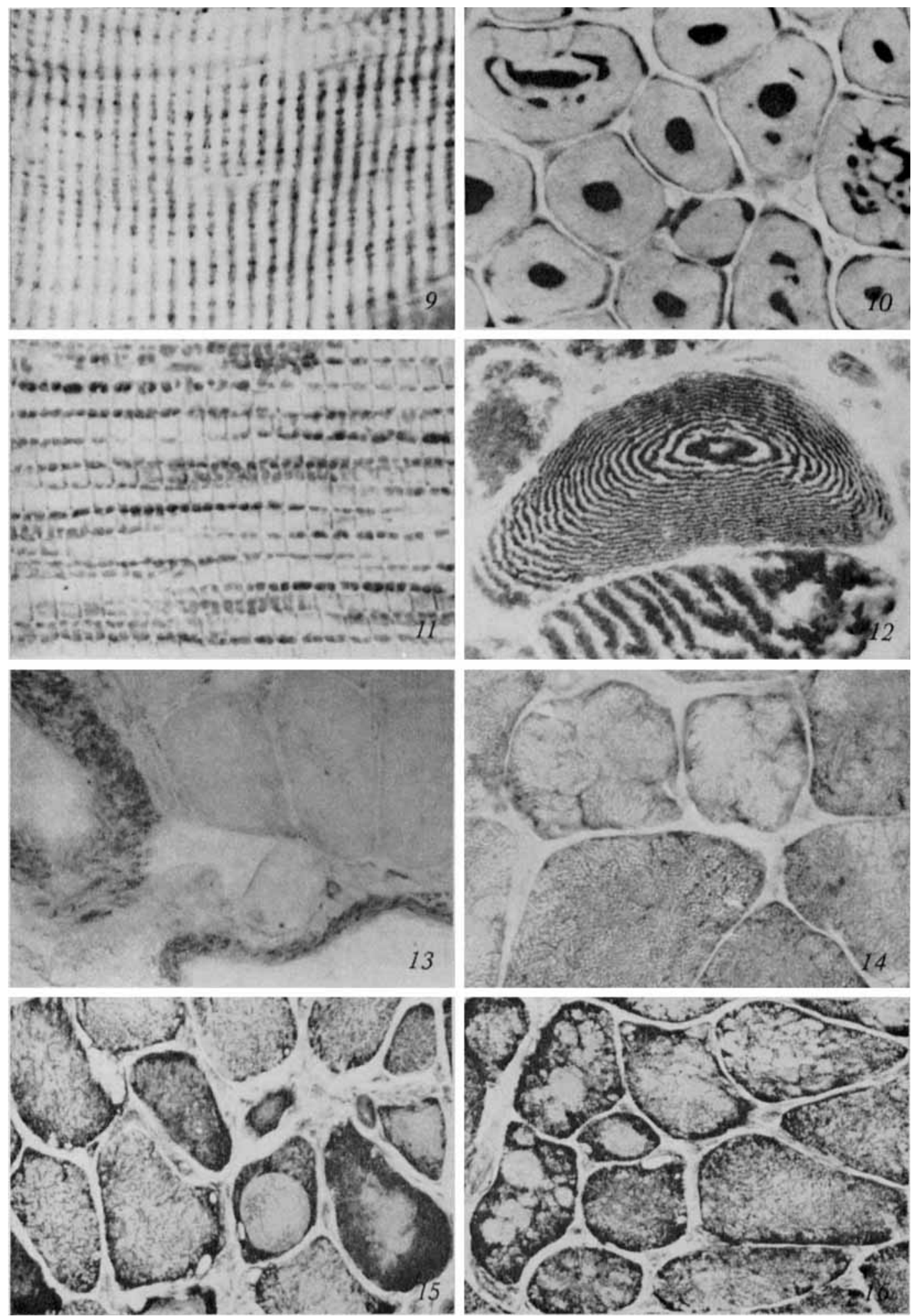
1. Complete absence of phosphorylase $a$ and $b$ :
a. Juvenile type $\mathrm{e}^{37-45}$
b. Later adult type ${ }^{46}$

2. Complete absence of phosphorylase $a$, severe impairment of phosphorylase $b$ :
a. Central core disease ${ }^{\mathbf{} 7}$

D. Central core disease. This condition is a "congenital nonprogressive myopathy." It, too, is associated with a generalized biochemical abnormality of skeletal muscle phosphorylase, shown by Mahler, ${ }^{+7}$ in addition to focal abnormal regions or "cores" in the skeletal muscle fibers, as originally described by Shy and Magee. ${ }^{\text {ts }}$ The generalized phosphorylase defect was not detected histochemically but probably would have been had the pertinent variant of the phosphorylase reaction been done, that is, with the omission of adenylic acid to study phosphorylase $a$ by itself. However, the cores were shown cytochemically1,19,47 to be virtually devoid of mitochondrial oxidative enzyme activity, of sarcoplasmic total phosphorylase $(a+b)$ activity and preformed glycogen, and of argyrophilic intermyofibrillar material (figures 17, 18). This suggested an absence from the cores of mitochondria, aqueous sarcoplasm, and the transverse component of the sarcoplasmic reticulum. Electron-microscopic observations by H. E. Huxley ${ }^{47}$ and by T. Wanko ${ }^{40}$ were in good agreement. They found that in the cores there was virtually complete absence of mitochondria and a severe reduction of other intermyofibrillar material. Speculation regarding the pathogenesis of the central core regions has been presented elsewhere. ${ }^{47}$

E. Muscle target fibers. Following denervation, a skeletal muscle fiber undergoes either a "concentric" (figures 19-25) or a "diffuse" (figures 22, 24) type of cytoarchitectural change. ${ }^{30}$ The "target" fiber (figures 19-24), so named because of its appearance on crosssection, ${ }^{7}$ manifests a concentric morphologic and cytochemical change. It is detectable with ordinary histologic technics but is better displayed with cytochemical methods. The typical target fiber has 3 fairly distinct concentric zones which extend along the length of the affected fiber. The zones are numbered

Fig. 17. Holmes silver stain for the transverse component of sarcoplasmic reticulum, fixed, $1400 \times$. Central core disease. The argyrophilic material is in the intermyofibrillar regions. In the peripheral, noncore (N-C) portion of the fiber the appearance is normal. Within the central core $(C)$, the amount of stained material is reduced, but at the core noncore junction it is increased.

Fig. 18. Same specimen as in figure 17, longitudinal section through the edge of a core (C), polarized light, $1400 \times$. Most of the argyrophilic material in the noncore region lies at the junction between bright A-bands and the dark I-bands, but a faint amount is at the level of the Z-bands. In the core, of which only the edge occurs in this section, the staining is excessive and somewhat disorganized, but the cross-banding is preserved.

Fig. 19. Phosphotungstic acid hematoxylin, fixed, $225 \times$. Denervation. Numerous 3-zoned target fibers are present.

Fig. 20. Ordinary Gomori trichrome, fixed, longitudinal section, polarized light, $560 \times$. Denervation. Two target fibers are present. The dark staining of the longitudinal strands of myofibril-like material in the central zone 1 masks their anisotropism.

Fig. 21. Modified trichrome, $560 \times$. Paramyotonia congenita, during an attack of flaccid paresis. A typical target fiber is present on the left, along with a hydropic, "vacuolated" fiber on the right.

Fig. 22. Succinate dehydrogenase, $560 \times$. Denervation. A 3-zoned target fiber (upper right) is adjacent to a large, normal fiber (lower left). The other 2 fibers show the diffuse type of change following denervation.

Fig. 23. Phosphorylase, $560 \times$. Denervation. A 3-zoned target fiber. In the peripheral zone 3 , the activity, which is in the intermyofibrillar region, generally has the normal pattern of localization.

Fig. 24. Myofibrillar A-band ATPase, $225 \times$. Denervation. Several 3-zoned target fibers are present. Some small fibers (dark and light) show the diffuse type of cytoarchitectural change. 

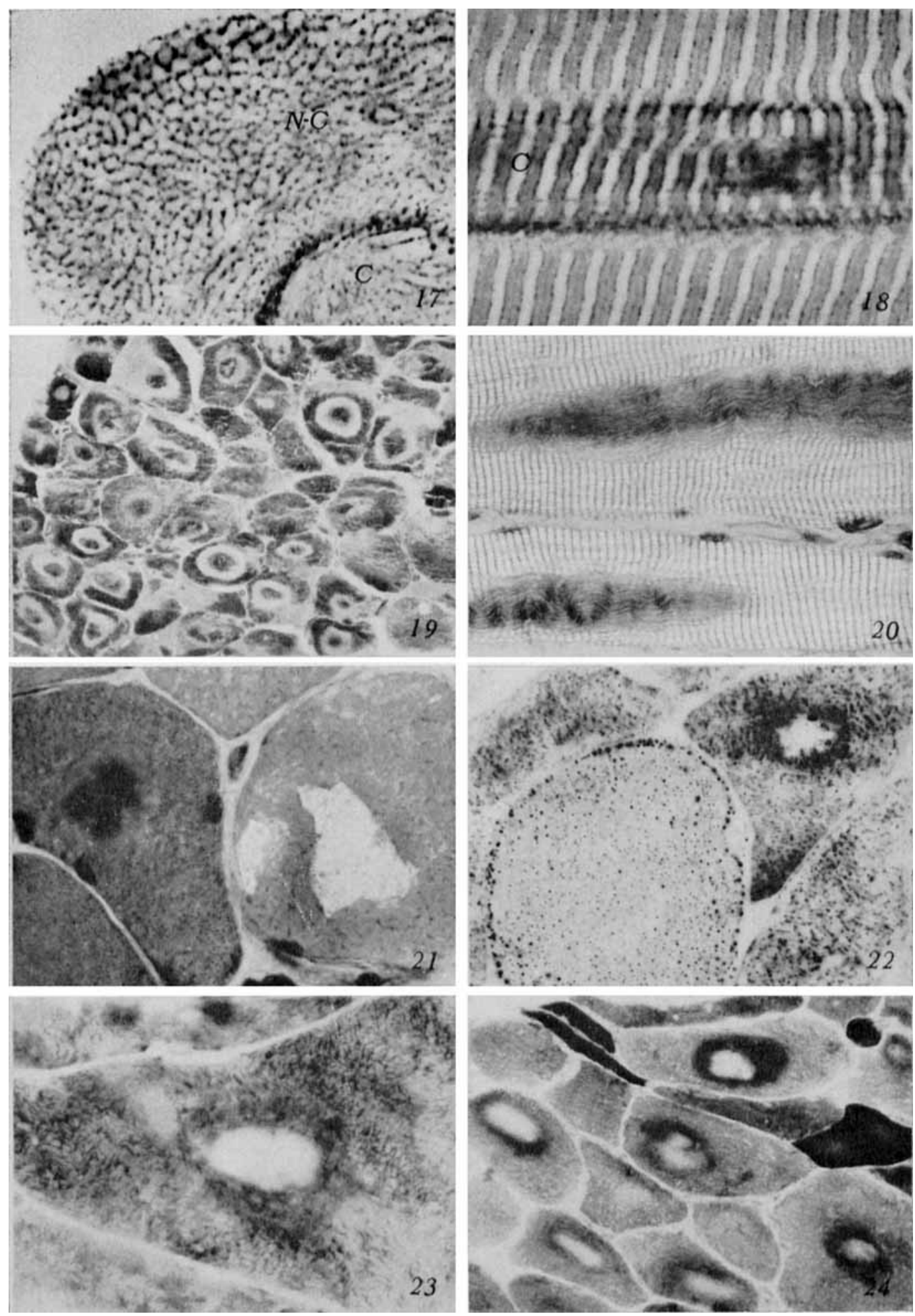
1,2 , and 3 from the center outward. ${ }^{7}$ Zone 1 consists of compact, longitudinally running strands of myofibril-like material, which lack cross-striations (figure 20), are uniformly anisotropic, and lack myofibrillar A-band ATPase activity (figure 24). There is virtually complete absence of mitochondrial oxidative enzyme activity (figure 22) and of the sarcoplasmic reactions (preformed glycogen and phosphorylase [figure 23]). Zone 2 has a reduced concentration of myofibrillar material, but a faint normal cross-striated pattern is seen in polarized light. Myofibrillar A-band ATPase activity is usually increased (figure 24). The mitochondrial oxidative enzyme activity is usually decreased, but sometimes it is increased (figure 22). The preformed glycogen and phosphorylase may be increased (figure 23) or decreased. Zone 3 is more nearly normal, but may show a mild diffuse change like that of denervated fibers which manifest only the diffuse type of cytoarchitectural abnormality.
Also present may be some atypical "targetoid" fibers which lack the intermediate zone 2 but probably still represent the same general concentric type of cellular reaction (figure 25).

It is only the typical target fiber which is of diagnostic significance. The typical target fibers have not been found in normal muscle or in muscle showing only myopathic changes; however, they were present in at least 60 per cent of the biopsies showing denervation, associated with a variety of diseases, in 80 patients. In addition, typical target fibers (figure 21) were found recently in biopsies from 3 of 5 patients having hypokalemic familial periodic paralysis and 2 of 3 patients having periodic paralysis associated with paramyotonia congenita, especially during the paralytic attack. It has been suggested ${ }^{7}$ that, in the majority of cases, the target appearance represents a reaction of the muscle fiber to denervation. It is probably an early reaction, because it often appears in fibers of normal diameter.

Fig. 25. Succinate dehydrogenase, $225 \times$. Denervation. Two-zoned targetoid fibers.

Fig. 26. Modified trichrome, $1400 \times$. Myotonic dystrophy. In part of the fiber near an internal nucleus, 8 cytoplasmic bodies without prominent shells are seen.

Fig. 27. Modified trichrome, $1400 \times$. Denervation. A typical 3-layered cytoplasmic body complex is in the center of an atrophied fiber.

Fig. 28. Phosphorylase, $560 \times$. Myotonic dystrophy. Several unstained cytoplasmic bodies are present.

Fig. 29. DPN-linked lactate dehydrogenase, $560 \times$. Denervation. Several unstained cytoplasmic bodies are present.

Fig. 30. Ordinary Gomori trichrome, longitudinal appearance, fixed, about $1600 \times$. Cultured chick embryo skeletal muscle. A cytoplasmic body is adjacent to a nucleus.

Fig. 31. Ordinary Gomori trichrome, longitudinal appearance, fixed, about $1600 \times$. Cultured chick enbryo skeletal muscle. Two cytoplasmic bodies are present near a nucleus.

Fig. 32. Myofibrillar A-band ATPase, $1400 \times$. Myotonic dystrophy. The normal, central part of the fiber is separated from the sarcoplasmic mass $(M)$ by the striated annulet $(A)$. In the central portion of the fiber, the darkly stained cross-sectioned myofibrils are separated by an unstained intermyofibrillar network. In the annulet, the longitudinally sectioned myofibrils are stained only in the A-bands. The sarcoplasmic mass has no activity.

Fig. 33. Phosphorylase, $560 \times$. Myotonic dystrophy. In each fiber, the peripheral sarcoplasmic mass $(M)$ is separated from the central normal part of the fiber by a striated annulet (A). The longitudinally sectioned annulets contain activity at the level of the I-bands.

Fig. 34. PAS for preformed glycogen, $560 \times$. Myotonic dystrophy. In the 3 affected fibers, peripheral sarcoplasmic masses $(M)$ are separated from the virtually normal central regions by striated annulets. The annulets (A) are sectioned longitudinally and contain glycogen at the level of the I-bands.

Fig. 35. Cytochrome oxidase, $560 \times$. Myotonic dystrophy. A large peripheral sarcoplasmic mass $(M)$ is present in one fiber. 

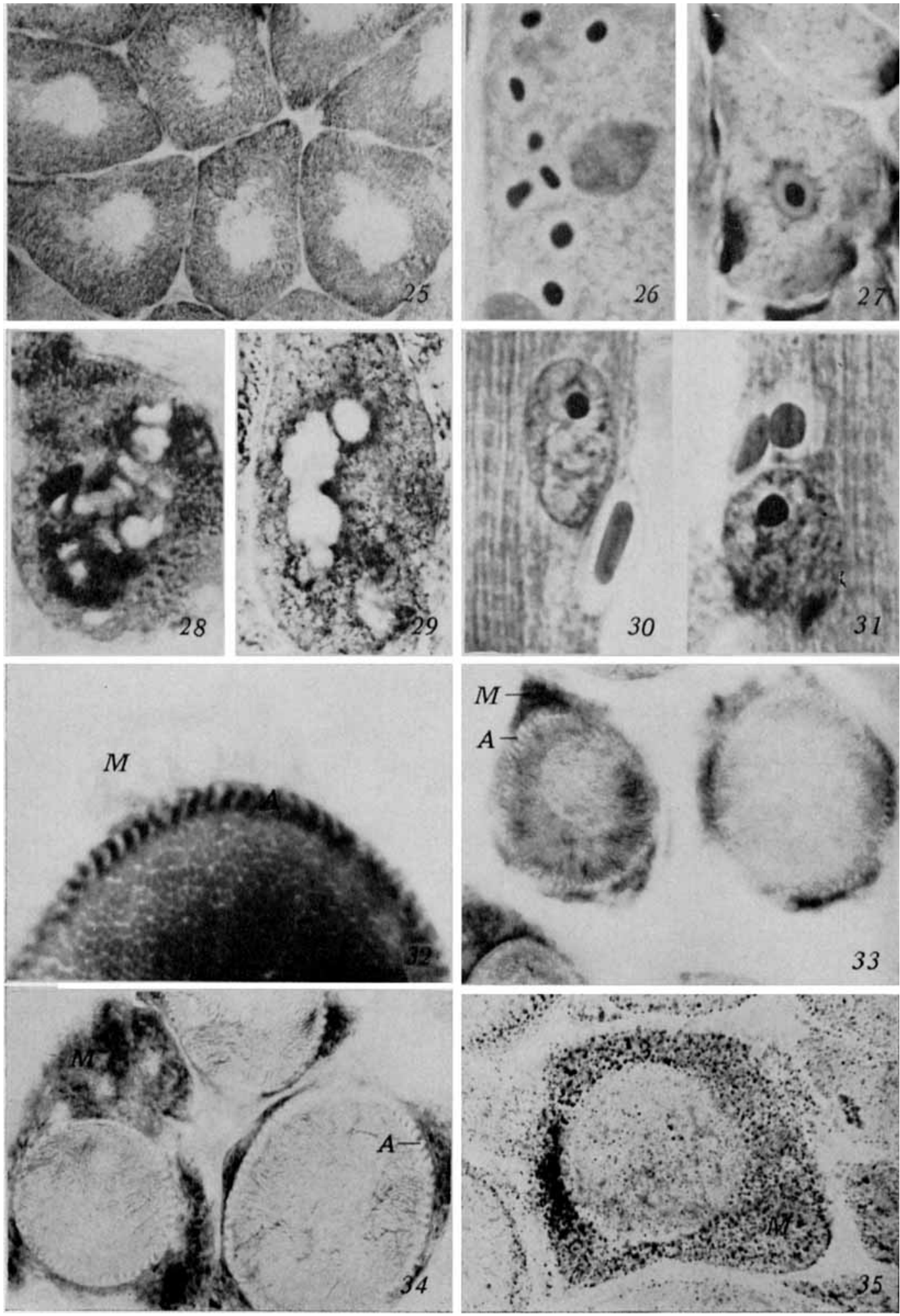
Whether the target fibers found in the periodic paralysis patients also are due to denervation, functional if not anatomic, is uncertain. (Although 1 affected patient of the paramyotonic type was diabetic and thereby could have had denervation, the other periodic paralysis patients with target fibers were not.) Some 2-zoned targetoid fibers occurred fairly often in human denervated muscle biopsies, and they have been described in experimentally denervated cat muscle by Tower; ${ }^{51}$ but, because fibers of similar appearance were also occasionally found in biopsy specimens which were purely myopathic, they are not of diagnostic significance.

Target and targetoid fibers may be distinguished from the somewhat similarly appearing central core fibers by the following criteria:

1. The cross striations of the myofibrils in the central zone of the target and targetoid fibers are lost, but they are preserved in the central cores.

2. The target, but not the targetoid, fibers have 3 concentric zones, while the central core fibers have only 2 with all stains except 1 (they may have 3 zones with the silver stain for sarcoplasmic reticulum [figure 17]).

3. Target and targetoid fibers are usually associated with other evidence of muscle denervation, while central core fibers are not. There may be rare normal-sized fibers undergoing degeneration in central core disease. ${ }^{47}$
F. Cytoplasmic bodies. Abnormal structures which may be termed "cytoplasmic bodies"52 have been found in pathologic human skeletal muscle fibers and in chick embryo skeletal muscle fibers grown in tissue culture. In human muscle fibers, the complete cytoplasmic body complex is spheroid and consists of 3 concentric portions-namely, the central "body proper," the intermediate "halo," and the outer "shell" (figure 27)-within the more nearly normal part of the affected muscle fiber. Cytochemical analysis suggests that the body proper is altered myofibrillar material which may or may not have retained its myofibrillar A-band ATPase activity, the halo is very dilute sarcoplasm which does not retain mitochondrial or sarcoplasmic reactions during histochemical incubations (figures 28, 29), and the shell is condensed intermyofibrillar material (figures 27-29).

The cytoplasmic bodies are uncommon, but they have been found in denervated muscle fibers in more advanced stages of atrophy (figures 27,29 ). Usually they occur less frequently in myopathic fibers, which are in moderate to advanced stages of degeneration; however, they are rather common in myotonic dystrophy (figures 26, 28). The cytoplasmic bodies are indicative of muscle fiber abnormality but are not of specific diagnostic significance. They are not to be taken as evidence of a viral infection. The zoning of the cytoplasmic body complex bears some similarity to that of the target fiber.

Fig. 36. Modified trichrome, longitudinal section, $1400 \times$. New myopathy with rod-shaped structures. In one portion (upper) of the fiber short rods appear in palisades. In a less affected region (middle) a few rods are parallel to the normal myofibrils (lower).

Fig. 37. Modified trichrome, $560 \times$. New myopathy with rod-shaped structures. Portions of some fibers show numerous rod forms, while other fibers are unaffected.

Fig. 38. Myofibrillar A-band ATPase, phase contrast to enhance definition, $1400 \times$. New myopathy with rod-shaped structures. The rods are unstained but the regions between them show activity. In the normal portion of the fibers, the myofibrils are stained, but the regions between them are not.

Fig. 39. PAS for preformed glycogen, $1400 \times$. New myopathy with rod-shaped structures. There is glycogen between the normal myofibrils in the lower part of the fiber and between the rocls in the upper part of the fiber, but not in the rods.

Fig. 40. Hematoxylin-eosin, phase contrast, $510 \times$. New myopathy with rod-shaped structures. Some fibers (marked $p$ ) contain numerous rods, and others (marked $o$ ) have none.

Fig. 41. Phosphorylase, $510 \times$, serial section to one in figure 40 . The fibers $(p)$ containing rods have high phosphorylase activity, and the fibers (o) with no rods are low in phosphorylase. 
Chick embryo skeletal muscle fibers, which. are innervated at 9 to 11 days in ovo,, 53 were removed for culture after 13 days in ovo. Hence they were denervated by the explantation procedure and then grown in vitro without nerve cells being present. ${ }^{54,5 \pi, 24}$ The cytoplasmic bodies in the cultured muscle fibers (figures 30,31 ) were morphologically and cytochemically similar to the bodies in the human denervated muscle fibers and thus are considered to be analogous. 52

G. Striated annulets and sarcoplasmic mass-
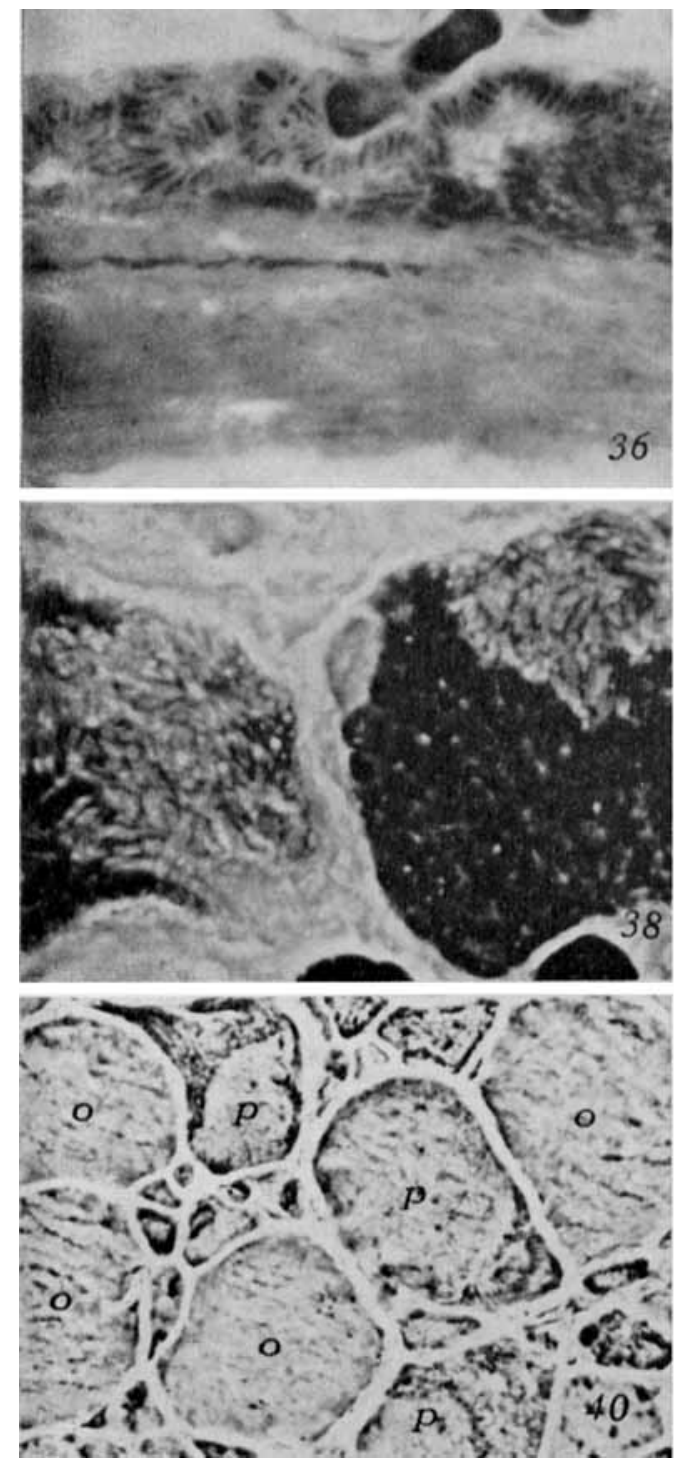

es in myotonic dystrophy. Heidenhain ${ }^{56}$ described Ringbinden ("striated annulets"57) and Wohlfart ${ }^{57}$ noted the presence of sarcoplasmic masses. These are the 2 most characteristic cytopathologic changes in myotonic dystrophy, ${ }^{58}$ but by some authors they have been confused one with the other and deemed "contraction band artifacts caused by acid fixatives." 59 That each change is distinct and not artifactual is demonstrated by careful histologic studies. ${ }^{58}$ Strong supportive evidence is given by cytochemical studies of fresh frozen sections, ${ }^{, 0}$ in
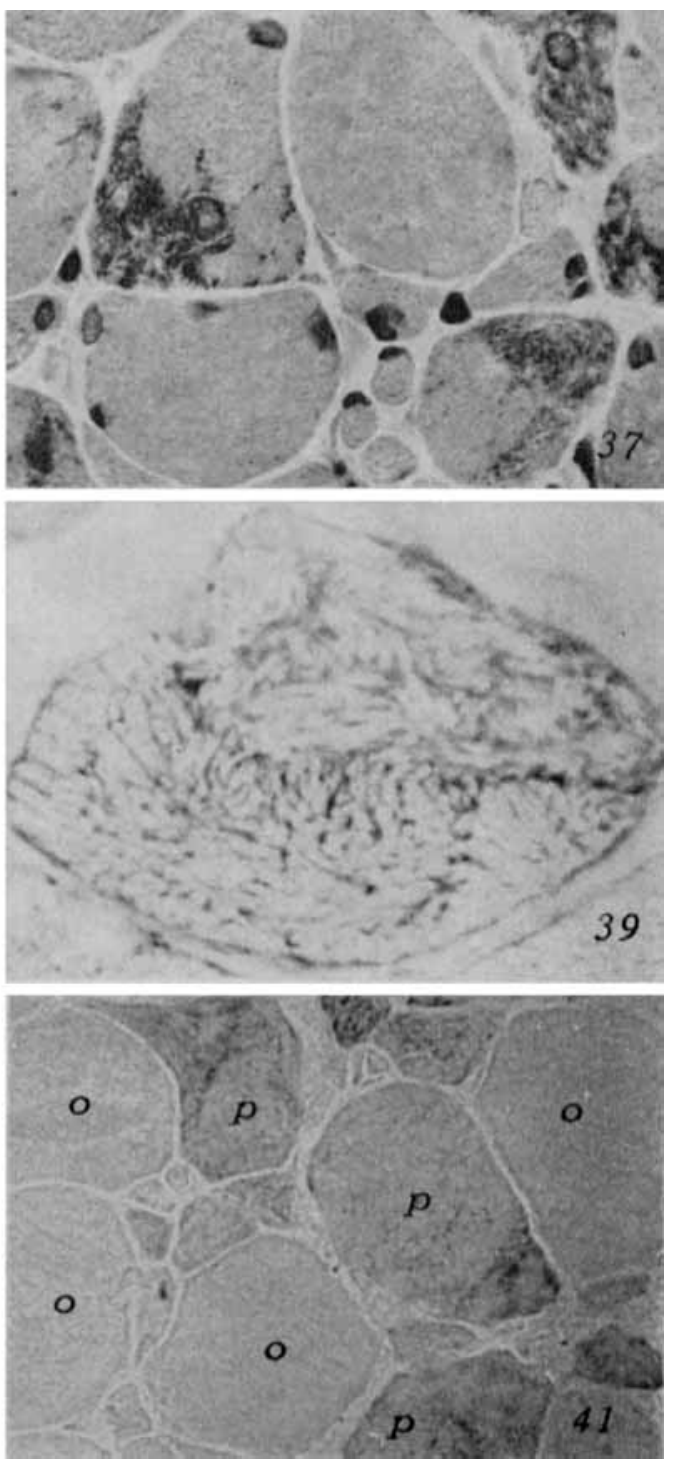
which the architecture of the unfixed tissue is very well preserved.

The striated annulets were found ${ }^{00}$ to be subunits of the muscle fiber, disoriented en $b l o c$ in respect to the rest of the muscle fiber. Thus when the muscle fiber is cut in cross section, the annulets may appear in longitudinal section (figures 32-34.) Within the annulets, the normal cytochemical activity, architecture, and interrelationship of the components were preserved, as demonstrated by the reactions for myofibrillar A-band ATPase (figure 32), for mitochondrial oxidative enzymes, and for sarcoplasmic phosphorylase (figure 33) and preformed glycogen (figure 34). It is not known whether the annulets were formed en bloc.

The sarcoplasmic masses ${ }^{60}$ lack myofibrillar A-band ATPase activity (figure 32). They consist of diffuse, disorganized intermyofibrillar material-namely, glycogen (figure 34), phosphorylase (figure 33), and mitochondrial oxidative enzymes (figure 35 ). The concentration of the intermyofibrillar material in the masses is probably equal to that elsewhere in the normal intermyofibrillar regions of fiber. Such material appears excessive because it is not separated into a network by interspersed myofibrils. The sarcoplasmic masses seem to be regions of the fiber in which the myofibrillar degeneration is greater than that of the intermyofibrillar material.

H. A new myopathy with rod-shaped structures. A recently studied 4-year-old white female who had a nonprogressive proximal muscle weakness present since birth was found to have skeletal muscle with unique cytopathology. (Full details of this case will be reported elsewhere.61) The present concern is with 2 features displayed by cyto- and histochemical technics: (a) the unique chemomorphologic change and (b) the apparently selective involvement of one histochemical type of muscle fiber.

In many muscle fibers, there were focal abnormal regions which extended for a considerable distance along the length of the fiber. In fresh frozen sections, within the abnormal regions there appeared short rod-shaped structures about $0.3-0.7 \mu$ in diameter and 1.5-5.0 $\mu$ in length. These rods were arranged irregularly or in palisades (figures 36-41), usually without respect to any axis of the muscle fiber, although in the minimally affected regions single rods were parallel with the adjacent normal myofibrils (figure 36 ). With the modified trichrome technic, the rods stained like altered myofibrillar material, and they did not have myofibrillar ATPase activity (figure 38). They were, therefore, histochemically like the altered myofibrillar material in zone 1 of the targets and like the body proper of some cytoplasmic body complexes. Interestingly, in the abnormal regions there was some ATPase activity between the rods (figure 38), but none was present in the intermyofibrillar regions of the apparently normal parts of the affected muscle fibers. There was intermyofibrillar material-mitochondrial oxidative enzymes and sarcoplasmic preformed glycogen (figure 39) and phosphorylase-between the rods, but it was somewhat less prominent than that occurring in the intermyofibrillar regions of the more normal parts of the affected fibers.

The unique focal collections of rods were prominent in some fibers but completely absent from others (figures 37, 40, 41). All of the affected fibers were of the type with high phosphorylase activity in their normal regions (figure 41), and all of the apparently unaffected fibers were of the low phosphorylase type.

\section{POSSIBLE MECHANISMS OF PATHOGENESIS}

A. Anatomical arrangement. Following denervation, muscle fibers belonging to the same motor unit (that is, those innervated by the same motor neuron) undergo atrophy together and are located in groups. By contrast, myopathic conditions in the early stages are associated with degeneration of only certain scattered muscle fibers without any obvious relation to motor units. ${ }^{58}$ To further study these 2 types of pathogenic processes, it is important to know whether or not all muscle fibers belonging to the same motor unit are of the same metabolic type, as defined histochemically. However, this point has not yet been established, although it may eventually be possible to make certain pertinent inferences from studies of human pathologic skeletal muscle.

The diagram shows the 2 possible arrangements of muscle fibers in a normal motor unit. In the first example, each nerve cell inner- 

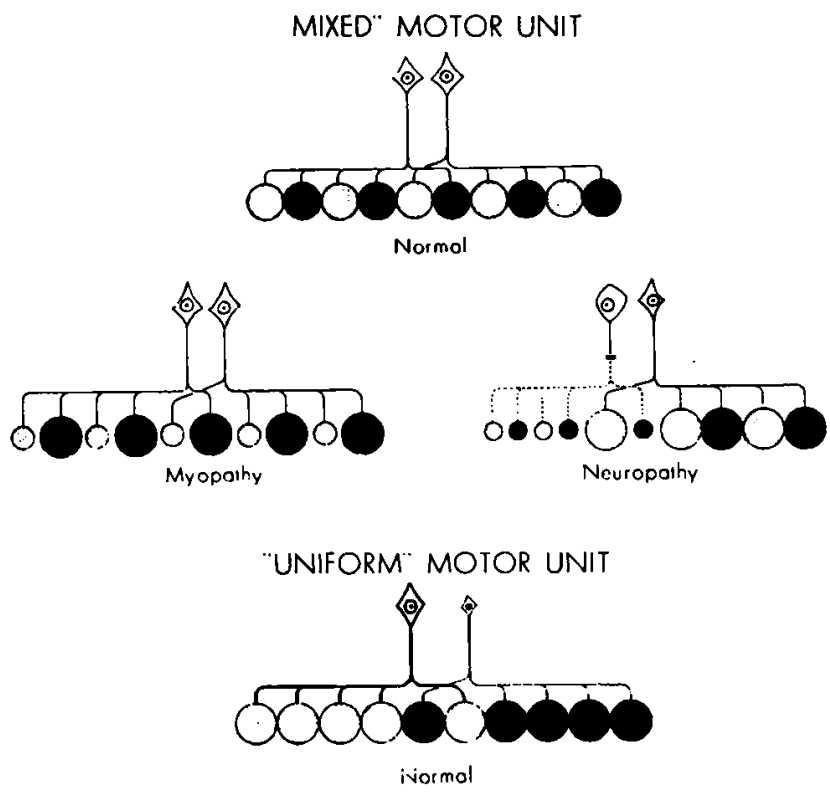

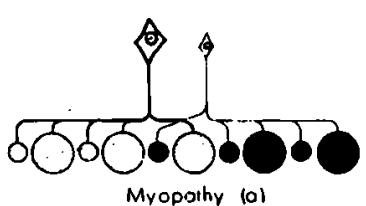

Myopothy (a)

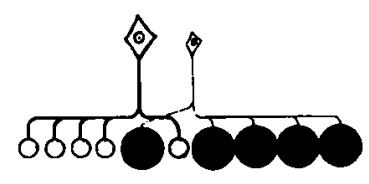

Myopolhy (b)

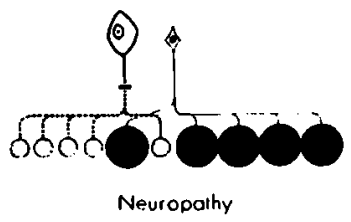

vates more than one histochemical type of muscle fiber, resulting in a "mixed unit." In the second example, each nerve cell innervates muscle fibers of only one histochemical type, resulting in a "uniform unit."

B. Myopathic conditions. We do not know the factor(s) which determines the selective involvement of scattered muscle fibers in a myopathy. Perhaps it is the metabolic differences between the fibers (such as can be demonstrated histochemically) which determine that some of them are preferentially affected in a given kind of myopathic process. In order for this mechanism of involvement to produce the scattered histological pattern of a myopathy, the motor unit would have to be of the mixed type (see text diagram, mixed unit, myopathy); for if the motor unit were uniform, this mechanism would produce a grouped pattern (see text diagram, uniform unit, myopathy $b$ ) indistinguishable from the grouped pattern expected in denervation of a uniform unit (see text diagram, uniform unit, neuropathy). Further, given this pathogenic mechanism of a myopathy, different proportions of the most susceptible type of muscle fiber in different muscle groups might determine the characteristic clinical distribution of various myopathic diseases.

The first definite example of a condition affecting preferentially one histochemical type of muscle fiber is the new myopathy with rodshaped structures. It is anticipated that further elaboration of this observation, which was possible only with histo- and cytochemical technics, might provide insight into the biochemical pathogenesis of the disease. Similar clues may reasonably be hoped for in further histo- and cytochemical studies of other myopathic diseases. Of interest is the recent report $^{62}$ that an abnormal (fetal type) myoglobin is present in the muscles of patients with muscular dystrophy of the pseudohypertrophic and the facio-scapulo-humeral types. An attempt to demonstrate myoglobin histochemically ${ }^{63}$ by its benzidine-peroxidase activity 
showed the highest concentration of that activity to be in the muscle fibers of Type $I$. If abnormal myoglobin is related to the pathogenesis of muscular dystrophy, perhaps those muscle fibers which normally have the greatest (or possibly the least) concentration of myoglobin degenerate earliest.

The other possibility is that the selective involvement of muscle fibers in certain myopathic processes is not related to histochemically demonstrable differences between muscle fibers. If so, the scattered pattern of fiber involvement in such conditions could occur in either mixed or uniform units (see text diagram, uniform unit, myopathy $a$ ).

C. Neuropathic conditions. Whether a motor unit is mixed or uniform is also of interest in relation to normal innervation of muscle fibers and to denervation. If the motor units are of the uniform type, specific influences of different metabolic varieties of motor neurons in the differentiation of the metabolically different muscle fibers must be considered. (Of interest are the pertinent studies of the neural influence of the differentiation of "fast" and "slow" muscles by Buller et al."64.65). Consequently, if it is found in denervated muscle associated with a given clisease that a certain histochemical type of muscle fiber is selectively involved, it may be a reflection of selective involvement of a certain metabolically distinct type of motor neuron.

If motor neurons determine the uniformity of muscle fibers in a given motor unit, and the differences of fibers in different motor units, another consequence is possible. There mav be an influence of neurogenic factors in seemingly "myopathic" disorders. For example, in central core disease, it was found 1.47 that there was an apparent lack of differentiation into the histochemically distinct types of muscle fibers. Moreover, central core fibers are somewhat similar cytochemically to denervated target and targetoid fibers. To be considered is the possibility that in central core disease there is an abnormality of the neural influence during embryonic differentiation of the muscle fibers. That is, defective neural factors may fail to cause differentiation of histochemically distinct types of muscle fibers and may permit abnormal regions (cores) to develop within the muscle fibers. Again, if uniform motor units are present, a second example of an unsuspected neurogenic influence might be any "myopathv" in which a certain histochemically defined variety of muscle fiber is preferentially affected, as in the new myopathy with rodshaped structures. If, with uniform motor units, the motor neurons do determine the differentiation of muscle fibers into different histochemical types, the selective involvement in the new myopathy with rod-shaped structures of fibers high in phosphorylase possibly might be a reflection of their abnormal development due to a defective neural influence.

To clarify the various pathologic mechanisms suggested, more studies are necessary.

\section{SUMMIARY}

Evidence has been presented to substantiate the claim that histo- and cytochemical studies are essential in the investigation of neuromuscular disease, based on what these technics have demonstrated to date and what they promise to disclose in the near future. In particular, 6 examples of cytopathologic changes in human skeletal muscle were consideredthe later adult type of phosphorylase defect; central core disease; muscle target fibers; cytoplasmic bodies; striated annulets and sarcoplasmic masses in myotonic dystrophy; and a new myopathy with rod-shaped structures.

The author is indebted to the following persons: Dr. G. Miltun Shy for kindling the interest in neuromuscular disease and for permission to study muscle biopsy material from his patients; Dr. Hibbard Williams for the biochemical analysis of phosphorylase activity; and Mr. Guy Cunningham for the technical assistance.

\section{REFERENCES}

1. Dunowitz, v., and PEARse, A. G. E.: Oxidative enrymes and phosphorylase in central core disease of muscle. Lancet 2:23, 1960.

2. vUBOwItz, v., and PEARSE, A. c. E.: Enzymatic activity of normal and dystrophic human muscle: a histochemical study. J. Path. Bact. 81:365, 1961.

3. INNAl, D.: Functional differentiation of skeletal mus cles. Acta Med. Okayaina 14:159, 1960.

4. BECKETT, E. B., and BouRNE, G. H.: Histochemistry of skeletal muscle and changes in some muscle diseases, in Structure and Function of Muscle, Bourne, G. H. cd., vol. III. New York, Academic Press, 1960, pp. 276-320.

5. BOUANE, G. H., and GOLARz, M. N.: Human muscular dystrophy as an aberration of the connective tissue. Nature 183:1741, 1959.

6. GOLARZ, M. N., BOURNE, G. H., and RuchaRdSON, H. D. Histochemical studies on human muscular dystrophy. J. Histochem. Cytochem. 9:132, 1961.

7. ENGEL, w. K.: Muscle target fibers, a newly recognized sign of denervation. Nature 191:389, 1961.

8. ENGEL, w. K., and MUMENTHaIER, M.: Cyto- and histochemistry of normal human skeletal muscle. To be published.

9. engel, W. K., and cunningham, G. G.: Cytological 
demonstration of myofibrillar and intermyofibrillar structures of striated muscle by a modified trichrome technique for fresh frozen sections. To be published.

10. NACHLAs, M. M., TSOU, K. C., DE SOUZa, E., Cheng, c. S., and seligman, A. M.: Cytochemical demonstration of succinate dehydrogenase by the use of a new p-nitrophenyl substituted ditetrazole. J. Histochem. Cytochem. 5:420, 1957.

11. NOVIKOFF, A. B., SHIN, w.-Y., and DRUCKER, J.: Mitochondrial localization of oxidative enzymes: staining results with two tetrazolium salts. J. Biophys. Biochem. Cytol. 9:47, 1961.

12. HESS, R., SCARPELLI, D. G., and PEARSE, A. C. E.: The cytochemical localization of oxidative enzymes. II Pyridine nucleotide-linked dehydrogenases. J. Biophys Biochem. Cytol. 4:735, 1958.

13. WATTENBERG, $x$. $w$., and LEONG, J. L.: Effects of coenzyme $Q_{10}$ and menadione on succinic dehydrogenasc activity as measured by tetrazolium salt reduction. J. Histochem. Cytocbem. 8:296, 1960 .

14. BURSTONE, M. s.: Histochemical demonstration of cytochrome oxidase with new amine reagents. J. Histochem. Cytochem. 8:63, 1960 .

15. Manual of Histologic and Special Staining Techniques. Armed Forces Institute of Pathology, Washington, D. C., 1957.

16. TAKEUCH, T.: Histochemical demonstration of branching enzyme (amylo-1,4 $\rightarrow 1,6$-transglucosidase) in animal tissues. J. Histochem. Cytochem. 6:208, 1958.

17. TAKEUCHI, T., and GLexNer, G. G.: Histochemical demonstration of uridine diphosphate glucose-glycogen transferase in animal tissucs. J. Histochem. Cytochem. 9:304, 1961 .

18. PADYKUDA, H. A., and HERMAN, E.: The specificity of the histochemical method for adenosine triphosphatase. J. Histochem. Cytochem. 3:170, 1955.

19. SHY, G. M., ENGel, W. K., and Wanko, T.: Central core disease. Ann. Int. Med. 56:511, 1962.

20. VERATT, E.: Investigations of the fine structure of striated muscle filer. J. Biophys. Biochem. Cytol. 10 in suppl. The Sarcoplasmic Reticulum, 1961, p. 1.

21. ANDERSSON-CEDERGREN, E.: Ultrastructure of motor end plate and sarcoplasmic components of mouse skeletal muscle fiber. J. Ultrastruct. Res., suppl. 1959. p. 1 .

22. PORTER, к. R.: The sarcoplasmic reticulum; its recent history and present status. J. Biophys. Biochem. Cytol. 10: in suppl. The Sarcoplasmic Reticulum, 1961, p. 219.

23. TUNIK, B., and HOLTZER, H.: The distribution of muscle antigens in contracted myofibrils determined by fluorescein-labeled antibodies. J. Biophys. Biochem. Cytol. 11:67, 1961.

24. ENGEL, w. K., and hoRvatr, B.: Myofibril formation in cultured skeletal muscle cells studied with antimyosin fluorescent antibody. J. Exp. Zool. 144:209, 1960.

25. ENGEL, w. K.: Cytochemistry of striated muscle: the toadfish swim bladder and the wasp fight muscle. To be published.

26. FAWCETT, D. W., and REveL, J. P.: The sarcoplasmic reticulum of a fast-acting fish muscle. J. Biophys. Biochem. Cytol. 10: in suppl. The Sarcoplasmíc Reticulum, 1961, p. 89.

27. sмiтн, D. s.: The structure of insect fibrillar flight muscle. A study made with special reference to the membrane systems of the fiber. J. Biaphys. Biochem. Cytol. 10: in suppl. The Sarcoplasmic Reticulum, 1961 , p. 123.

28. KNOLL, P.: Uber protoplasmaarme und protoplasmareiche Muskulatur. Denkschr. Akad. Wiss. Wien, math.-nat. Kl. 58:633, 1891.

29. KRüGER, P.: Tetanus und Tonus der quergestreiften Skelettmuskeln der Wirbeltiere und des Menschen. Leipaig. Akad. Verlagsges., 1952.

30. DENNY-BRoWn, D. E.: The histological features of striped muscle in relation to its functional activity. Proc. Roy. Soc. (London) ser. B 104:371, 1929.

31. Nachmas, V. T., and Padykula, H. A.: A histochemical study of normal and denervated red and white muscles of the rat. J. Biophys. Biochem. Cytol. 4:47, 1958.
32. GeoHGe, J. C., and NaIx, R. N.: Studies on the structure and physiology of the flight muscles of birds. The structure of the pectoralis major muscle of the pigeon in disuse atrophy. J. Animal Morph. Physiol. 6:95, 1959.

33. DUbowitz, v., and PEArse, A. c. E.: Reciprocal relationship of phosphorylase and oxidative enzymes in skeletal muscle. Nature 185:701, 1960.

34. DUBOWITZ, v., and PEARSE, A. G. E.: A comparative histochemical study of oxidative enzyme and phosphorylase activity in skeletal muscle. Histochemie 2: $105,1960$.

35. PEARSE, A. G. E.: Direct relationship of phosphorylase and mitochondrial alpha-glycerophosphate debydrogenase activity in skeletal muscle. Nature 191:504, 1961 .

36. HESS, R., and PEARSE, A. G. E.: Dissociation of uridine diphosphate glucose-glycogen transglucosylase from phosphorylase activity in individual muscle fibers. Proc. Soc. Exper. Biol. \& Med. 107:569, 1961.

37. IC ARDLE, B.: Myopathy due to a defect in muscle glycogen breakdown. Clin. Sc. 10:13, 1951.

38. schmid, R., and мakLER, R.: Chronic progressive myopathy with myoglobinuria; demonstration of a glycogenolytic defect in the muscle. J. Clin. Invest. 38 $2044,1959$.

39. SChMid, R., Robrns, P. W., and TraUt, R.: Glycogen synthesis in muscle lacking phosphorylase. Proc. Nat. Acad. Sci. (USA) 45:1236, 1959 .

40. LARNEK, J., and villar-palasi, c.: Enzymes in a glycogen storage myopatby. Proc. Nat. Acad. Sci. (USA) 45:1234, 1959 .

41. Roseins, P. w.: Immunological study of human muscle lacking phosphorylase. Fed. Proc. 19:193, 1960.

42. SGHMID, H., and HAMMAKER, x.: Hereditary absence of muscle phosphorylase (McArdle's syndrome). New England J. Med. 264:223, 1961.

43. PEARSON, C. M., RUMER, D. G., and MOMMAERTs, w. F. H. M.: Defect in muscle phosphorylase: a newly defined human disease. Clin. Res. 7:298, 1959.

44. MOMMAAERT, w. F. H. M., ILLINGWORTH, B., PEARSON, C. M., GUillory, R. J., and SERAYDARIAN, K.: A functional disorder of muscle associated with the absence of phosphorylase. Proc. Nat. Acad. Sci. (USA) 45:791, 1959 .

45. PEARSON, G. M., RMMER, D. G., and MOMMAEXTs, w. F. H. MI.: A metabolic myopathy due to absence of muscle phosphorylase. Am. J. Med. 30:502, 1961.

46. ENGEL, $w$. K., and EYERMAN, E. L.: Skeletal muscle phosphorylase defect-an adult type presenting as a late onset myopathy. To be published.

47. ENGEL, w. K., FOSTER, J. B., hUGHES, B. P., HUXLEY, H. E., and Mahlak, R.: Central core disease-an investigation of a rare muscle cell abnormality. Brain $84: 167,1961$.

48. SHY, G. M., and MAGEE, K. R.: A new congenital nonprogressive myopathy. Brain 79:610, 1956.

49. SEITELBerger, F., waNko, T., and GAVIN, M. A.: The inuscle fiber in central core disease. Histochemical and electron microscopic observations. Acta Neuropath. 1:223, 1961 .

50. ENGEL, w. K., and MUMenthaler, M.: Cyto- and histochemistry of denervated human skeletal muscle. To be published.

51. TOWER, s. s.: Atrophy and degeneration in skeletal muscle. Am. J. Anat. 56:1, 1935 .

52. ENCEL, w. K.: Cytoplasmic bodies in pathologic human and cultured chick embryo skeletal muscle fibers. To be published.

53. MUMrenthalea, $M$., and maEI, $w$.: Cytological localization of cholinesterase in developing chick embryo skeletal muscle. Acta anat. $47: 274,1961$.

54. ENGEL, w. K.: Cytological localization of cholinesterase in cultured skeletal muscle cells. J. Histochem. Cytochem. 9:66, 1961 .

55. ENGEL, w. X.: Cytological localization of glycogen in cultured skeletal muscle. J. Histochem. Cytochem. 9:38, 1961.

56. HEIDENhaiN, M.: Uber progressive Veränderungen der Muskulatur bei Myotonia atrophica. Beitr. z. path. Anat. u. z. allg. Pathol. 64:198, 1918.

57. WOHLFART, G.: Dystrophia myotonica and myotonia 
congenita. Histopathologic studies with special reference to changes in the muscles. J. Neuropath. Exper. Neurol. 10:109, 1951.

58. GREENFIELD, J. G., SHY, G. M., ALVORD, E. C., and berc, $\tau$. : An Atlas of Muscle Pathology in Neuromuscular Discase. Edinburgh, E. \& S. Livingstone, 1957.

59. ADAMIS, R. D., DENNY-BROWN, D., and PEARSON, C. M.: Disenses of Muscle. A Study in Pathology. New York, Paul B. Hoeber, 1954, p. 462.

60. ENGEL, $\mathbf{w}$. K.: Chemocytology of striated annulets and sarcoplasmic musses in myotonic dystrophy. J. Histochem. Cytochem. 10:229, 1962.

61. SHY, G. M., ENGEL, W. K., SOMERS, J. E., and WANKo, T.: To be published.
62. Whorton, C. M., HCDGNS, P. C., and CONNERs, J. J.: Abnormal spectrophotometric absorption spectnums of myoglobin in two forms of progressive muscular dystrophy. New England J. Med. 265:1242, 1961.

63. DREWS, G. A., and ENGEI, W. K.: An attempt at histochemical localization of myoglobin in skeletal muscie by the benzidine-peroxidase reaction. J. Histochem. Cytochem. 9:206, 1961.

64. HULLER, A. J., ECCLES, J. C., and ECCLES, R. M.: Differentiation of fast and slow muscles in the cat hind limb. J. Physiol. 150:399, 1960.

65. BULLER, A. J., ECCLES, J. C., and ECCLES, R. M.: Interactions between motoneurones and muscles in respect of the characteristic speeds of their responses. J. Physiol. 150:417, 1960. 


\section{Neurology}

\section{The essentiality of histo- and cytochemical studies of skeletal muscle in the investigation of neuromuscular disease \\ W. King Engel \\ Neurology 1962;12;778 \\ DOI 10.1212/WNL.12.11.778}

This information is current as of November 1,1962

\section{Updated Information \& \\ Services}

Citations

Permissions \& Licensing

Reprints including high resolution figures, can be found at:

http://n.neurology.org/content/12/11/77 8.citation.full

This article has been cited by 1

HighWire-hosted articles:

http://n.neurology.org/content/12/11/77 8.citation.full\#\#otherarticles

Information about reproducing this article in parts (figures,tables) or in its entirety can be found online at:

http://www.neurology.org/about/about_ the_journal\#permissions

Information about ordering reprints can be found online:

http://n.neurology.org/subscribers/adve rtise

Neurology ${ }^{\circledR}$ is the official journal of the American Academy of Neurology. Published continuously since 1951, it is now a weekly with 48 issues per year. Copyright (C) 1962 by the American Academy of Neurology. All rights reserved. Print ISSN: 0028-3878. Online ISSN: 1526-632X.

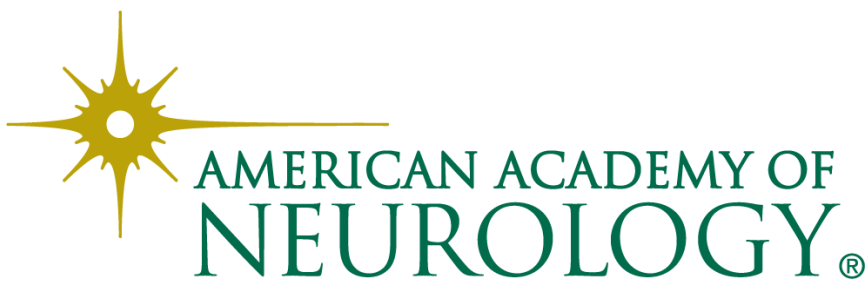

